\title{
FINE STRUCTURE OF THE RENAL GLOMERULUS
}

\author{
HIROSHI SAKAGUCHI \\ Department of Pathology, School of Medicine, \\ Keio University
}

(Received on August 1, 1955)

Normal structure of the glomerulus is closely related with the function of the kidney. Accordingly it goes without saying that the delineation of a normal structure of the renal glomerulus is requisite as a baseline for evaluation of pathologic alteration of the renal glomerulus.

This problem has long been the subject of study by many investigators without having yet been completely solved. This report is an observation of the renal glomerulus using a specific silver impregnation method (1951) (D. B. Jones) and electron microscopy.

\section{OBSERVATIONS ON THE GLOMERULAR BASEMENT MEMBRANE BY THE SPECIFIC SILVER IMPREGNATION METHOD}

Among the all structural elements of the glomerulus, the basement membrane might have the most intimate relation between the function of the glomerulus. Many interesting problems still remain to be studied in the structures of the glomerular basement membrane when it is compared with its function. In 1688 Malpighi discovered the renal glomerulus by means of injection through the renal artery. He considered it a gland which might possibly give the origin to the uriniferous tubule. Mall, 1896, after boiling renal tissue in water, digested frozen section with pancreatin and found a homogenous striped membrane still present in the glomerular loops. Rühle, 1897, also reported that the existence of a regularly stripted fibrous membrane showing numerous fine pores. As for the existence of the argylophil fibrils in the glomerular basement membrane, two different views have been existed quite opposite to each other. Volterra (1928), MaxClara (1932) described the existence of argylophil, net-like fibrils in the glomerular loops. However, Allen (1927), Zimmermann (1933), McGregor (1929), Möllendorff (1929, 1930) did not note the existence of them. Bargmann (1929) described the glomerular membrane as a deep black or brown line when impregnated with silver. Jones (1951) reported a specific silver impregnation method. Results obtained by this method he described the glomerular basement 
membrane as follow: the so-called basement membrane of the glomerular tuft is continuous at the hilus with the basement membrane of the parietal layer of Bowmans capsule, which in turn is continuous with the basement membrane of the proximal convoluted tubule.

\section{Methods and Materials}

The material used in the present study were some new-born and adult human post-mortem kidneys in which there were no marked pathological changes. Formalin fixed, paraffin imbedded material was used. All sections were cut as thin as 2 or $3 \mu$. H-E stain, Mallory azan stain, Pap's silver impregnation method, periodic acid-Schiff reaction and the specific silver impregnation method (the periodic acid silver reaction, Jones) were used in this study. Various impregnation methods have been used by many workers for the purpose of staining the glomerular basement membrane. Among others, the method recently reported by Jones, is particularly worthy of notice. Greater part of the observation of this report are the results obtained by this method.

Furthermore, the pathological change of the glomerular basement membrane were briefly studied about the cases of glomerulitis, subacute and chronic glomerulonephritis, focal glomerulonephritis, renal arteriosclerosis and wireloop lesion in disseminated lupus erythematosus.

\section{Observations}

a) Basement membrane and mesangium of normal glomerulus.

It is widely recognised that the basement membrane of the glomerular tufts are stained in blue by Mallory azan stain and in purple-red by periodic acid Schiff stain. However, by these routine staining it is very difficult to differenciate each components of the glomerulus particularly the relation between the basement membrane and the mesangium. Furthermore, by the Pap's silver impregnation method, some delicate argylophil fibrils were found in the hilus of the glomerulus, but no fibrils were demonstrated in the other part of it. The specific silver impregnation method (Jones) was considered to be the best one for studing the glomerular basement membrane.

In the glomerulus of children, the basement membrane of the glomerulus is constituted from two layers. The outer layer, deeply stained by the specific silver impregnation method, is continuous at the hilus with the parietal layer of Bowmans capsule, which is continuous with the basement membrane of the proximal convoluted tubule. There is no continuity between this basement membrane and the afferent and efferent arteriol. These facts above mentioned suggest that the basement membrane is anatomically related to the glomerular 
epithelium. The so-called mesangium (Zimmermann), the interstitial connective tissues of the glomerular tufts are demonstrated between the opposite two epithelial basement membrane. (Fig. 1) By the specific silver impregnation method, these mesangium are stained as dark brown area containing the blackstained nuclei of the so-called mesangium cells. In the normal adult kidney the wall of the capillary loops are so distended that the relation between the mesangium and basement membrane is not so clear, but this relation is clearly demonstrated in the glomerulus of the children. The relation will be confirmed by the electron microgram which is described later.

b) Pathological changes of the basement membrane of the glomerulas tuft.

In the inflammatory condition of the glomerular tuft, such as glomerulitis, glomerulonephritis, the basement membrane of the glomerular tuft divided into two or three rather delicate argylophil fibrils. (Fig. 2) This finding revealed as the thickness of the basement membrane by the H-E stain and the Mallory stain. At the same time leucocytic infiltration and inflammatory edema are presented in the glomerulus. Also fine argylophil fibrillar material, and increasing number of mesangium cell are demonstrated in the mesangium.

In the glomerulus of the subacute glomerulonephritis, these inflammatory changes are more strinkingly demonstrated than that of the acute stage. (Fig. 3) Usually some glomerular tufts show glomerular scarring and shrinkage. Also capsular epithelial proliferation is often striking. In the chronic glomerulonephritis, glomerular scarring may become severe as a result of acute and subacute inflammation of the glomerulus. Some scars suggest glomerular collaps and capillary obliteration with little connective tissue production. These scars are surrounded by a thick hyaline crescent suggesting that the end stage of the capsular epithelial proliferation. (Fig. 4) The basement membrane of these glomerulus were stained brown by the sepcific silver impregnation method. Another scars suggest the end stage of the connective tissue proliferation around the tufts. The glomerular basement membrane in these scars shows sweelling and degenration, and is stained brown or grey in color by the specific silver impregnation method.

In the glomerular lesion of the focal glomerulonephritis the basement membrane of this lesion were fragmented and by the specific silver impregnation method they were observed as black stained, frayed fibrils in greyish stained amorphous material. (Fig. 5)

On the contrary in the glomerular lesion of the renal arteriosclerosis, there is no inflammatory change such as fraying or fragmentation of the basement membrane, leucocytic infiltration, edema etc. Irregular increasing of argylophil 
fibrils around the glomerular tufts is the characteristic finding of the arteriosclerotic kidney by the specific silver impregnation method.

The glomerular alteration of wire-loop lesion in disseminated lupus erythematosus are of considerable interest and aid in the interpretation of the structural arrangement when it compared with the findings of the electron mecroscope. Three layers were observed in the basement membran of the glomerular tufts in wire-loop lesion: the process-like outer layr, the black-stained outer basement membrane and the rather delicate black stained inner basement membrane. In comparison with these findings to the electron microscope, it may be possible to identify the process-like outer layer in silver impregnation figure with the process-like structure in electron microgram. The inner basement membrane seems to be the endothelial layer which acquired the argylophility in pathological condition. The hyaline material which are stained in brown by silver impregnation method and in purple red by Mallory stain are demonstrated between the outer and inner basement membrane, in the mesangium and in the process-like structure.

In wire-loop lesion, the submicroscopic layer (inner translucent layer) may well be a primary site of the deposition of the hyaline material in the glomerulus. Hence the essential change of the wire-loop lesion in disseminated lupus erythematosus is not the fibrinoid necrosis of the glomerular tuft but the deposition of hyaline material in the glomerular tuft and mesangium.

\section{OBSERVATIONS OF THE GLOMERULUS WITH ELECTRON MICROSCOPE}

With a background of the light-microscopic findings, the submicroscopic structure of the normal glomerulus was studied with the electron microscope.

Since ultrathin section has become available for electron microscope, the fine structure of the glomerulus was reported by several investigators. In 1950, Pease, Baker and Warren illustrated certain basic structural features of the glomerular capillaries. At that time, they considered that a continuous layer of endothelial cytoplasm was not present and they described "in the most place the naked basement membrane is applied directly to the flowing blood stream".

Dalton considered the ridges of the basement membrane as the processes of the epithelial cells and also noticed a very delicate endothelial membrane internal to the basement membrane. Thus, he noticed three membrane between the capillary lumina and the Bowmans capsule space, i.e., a complete endothelial lining, a continuous basement membrane, and a layer former by the fine interdigitating processes of the cells of the visceral layer of Bowmans capsule. 
In 1953, Rinehart and his co-workers reported that the basement membrane proper was a derivative of the glomerular capillaries as a "complex of three intimately related specialized structure. (1) the luminal, delicate, highly porous submicroscopic layer, the "lamina fenestra" (lining network), (2) a thin, smooth, dense but probably finely porous membrane which must serve as an ultrafilter, the "lamina densa", and (3) an intricate external layer composed largely of characteristically shaped, rather uniform processes, the "pedicles", which are terminal extensions of secondary processes, trabeculae, from "podocyte" (epithelial) cytoplasm. In Pease's most recent study of the glomerular basement membrane, he observed a regularly arranged system of "pores" throughout the endothelial sheet. However, he was uncertain whether or not the pores were genuine structure or fixation artifact.

In Rinehart's most recent study of the glomerulus, he noticed a fine structural organisation on some sections of the basement membrane. Concerning the endothelial cell, he described that "the endothelial cytoplasm" is of variable and irregular thickness. In some places this layer is quite thin. In its thinner segments it often contains a series of small vesicles in the range of $200 \mathrm{~A}$ to $800 \mathrm{~A}$ in diameter."

Many obscure points of the fine structure of the glomerulus has been clarified one after another by these authors. However, the fact that "the connective tissue was not found in the normal glomerulus" was the only detail about the mesangium or interstitial connective tissue which is widely discussed among the pathologists.

The author intended to discuss also the subject of the mesangium.

\section{Material and Method}

This experiment performed with the kidney of dog. Small blocks of kidney from blood-letting, normal adult dog were fixed immediately in $1 \%$ buffered osmic acid. The osmic acid solution was buffered at about pH 7.2-7.4 with veronal acetate buffer (Palade). The specimen were fixed for three hours. After washing with distilled water and dehydrated in graded alcohols they were embedded in a mixture of n-butyl methacrylate ( 7 Parts) and methyl methacrylate (3 Parts). Sections about 0.1 micron in thickness were cut with a glass flagmented knife described by Kushida and a modified sliding microtom.

Some of these sections were examined without removal of the embedding medium.

\section{Observation}

There are four chief components of the glomerulus, the endothelium, the 
basement membrane, the epithelium, and the mesangium.

By the electron microscop, three layer could be observed in a wall of the glomerular tuft. The basement membrane, highly dense, is the middle layer of the wall. Inside of the basement membrane there is double-line structural, less dense, endothelial layer. Outside of the basement membrane, many processlike structure (the epithelial layer) were observed.

The basement membrane: The basement membrane is a continuous layer of highly density and having thickness of $100 \mathrm{~m}_{\mu}$. In some sections the basement membrane appears to have thickness of $200 \mathrm{~m} \mu$, in order to tangential sectioning of the capillary loop. However, a fibrillar structure or vesicles could not be able to observe.

The relation between the mesangium and the basement membrane: The basement membrane and process-like structure can be demonstrable between the mesangium and the Bowmans capsule space. However, between the mesangium and the capillary lumina of the glomerular tufts, there is neither basement membrane nor process-like structure. Only a thin layer of the protoplasm of the endothelial cell fixes the boundary between the mesangium and the capillary lumina. These finding was confirmed by the specific silver impregnation method and with electron microscope. (Fig. 1,8) There have been no detail about this problem, but this finding might have an important factor to solve the problems of renal diseases. A structure having a argylophil character by the specific silver impregnation method assumed to be the basement membrane that mentioned above. Both the process-like structure and the endothelial layer did not show any argylophil character except in their pathological condition.

The mesangium: The mesangium observed by an area of high density. In this field, the mesangium-cell and the other substances of high density were observed. The nuclei and the cytoplasm of the mesangium-cells were compacted with the 30 to $50 \mathrm{~m}_{\mu}$ granule. In the mesangium-cells some mitochondria were observed. However in order to high density the extracellular area of the mesangium is not possible to descern structural differentiation. But, no apparent fibrillar structure could be observed in these area. (Fig. 7)

The epithelial cell: The nuclei of the epithelial cells were compacted with fine dust like granule. The nuclear membrane is constituted from two layers, the inner layer having thickness of $20 \mathrm{~m} \mu$ and more thinner outer layer. Between the two layers, there is a clear demarcation of $10 \mathrm{~m}_{\mu}$. The cell membrane of the epithelial cell is $10 \mathrm{~m} \mu$ thickness. The epithelial cells have relatively pale cytoplasm which contain fine structural elements those found in most cells, i.e. mitochondria, canalicular structur of the endoplasmic reticulum and the Golgi 
area which was reported by Dalton and Sjöstrand. (Fig. 10)

The process-like structure exhibited a somewhat greater density than the epithelial cell. However this is not always so.

In the neighbourhood of the epithelial cells, foot-like processes of the epithelial cells attached to the basement membrane may exhibit a cytoplasm of low density. Some of the epithelial cells were laid over the two different capillary loops by their foot-like processes.

The processes or foot-like processes attached to the basement membrane commonly have a pyramidal form. Most of them exhibited gave the basis of $150 \mathrm{~m}_{\mu}$ to $2.5 \mathrm{~m}_{\mu}$ wide and their length were $0.2 \mu$ long. As the existence of the continuation and the similar construction between the process and the epithelial cells, it is evident that these processes were a peripheral cytoplasm of the epithelial cell. Also evident that the surface of the capillary loops were covered completely with the processes of the epithelial cells. The epithelial process abutting the basement membrane are sharply demarcated from it by a fine line of light density having thickness of $25 \mathrm{~m} \mu$. Between two process, there are distances of light density of about $50 \mathrm{~m} \mu$ wide.

The endothelial cell: The nuclear membrane of the endothelial cell having thickness of 20 to $40 \mathrm{~m} \mu$ and is constituted from two layers. The endothelial cells have ovoid nuclei. Most of the cytoplasm of the endothelial cell is of relative low density and processes the usual cellular components, i.e., mitochondria, canalicular structure of the endoplasmic reticulum, and small vesicles. The marginal cytoplasm of the endothelial cell revealed very thin film of the cytoplasm covering the inner aspect of the basement membrane. (Fig. 12) However many interruption existed in this layer. (Fig. 9) This endothelial layer is of variable and irregular thickness. In some places of this layer mitochondria can be observed. The thinnest part of this layer has the thickness of $50 \mathrm{~m} \mu$. Although the region covering over the mesangium, the endothelial layer exhibited thicker than the other part of it. (Fig. 8)

\section{COMMENT}

The fine structure of the glomerular tufts has been revealed by the many authors with histological or electron microscope. This report was performed by the comparative observation of the findings by the specific silver implegnation method and those with electron microscopy. In this paragraph three chief subjects, i.e. the basement membrane and the mesangium, the three dimentional patterns of the process-like structure of the epithelial cells, and the questionable 
interruption of the endothelial layer will be discussed.

The basement membrane and the mesangium:-By the specific silver impregnation method, no argylophil fibrils exist between the mesangium and the glomerular tufts. However in a small part of these boundering portion very delicate argylophil fibers could be observed. Moreover some glomerular capillaries were free from the mesangium. Thus it had been expected that some glomerular tufts were liberated from the mesangium, but in observation with electron microscope, no capillary tufts were liberated from the mesangium. Jones reported his own observation about the inflammation of the glomerulus as follows: In acute inflammation of the glomerulus, inflammatory cells and proliferated interstitial cells in the interstitial spaces are the major factor in the production of hypercellularity, rather than pure endothelial proliferation. It is generally accepted that the mesangium i.e. interstitial space, is the area of reaction. This suggestion is easily acceptable by the reasons as follows. That is the fact that only a thin film of the endothelial layer exist between the mesangium and capillary tuft, so the mesangium is easily influenced by the changes of the chemical components of the blood.

The three dimentional patterns of the layer in which innumerable processlike structure were found:-Because of the difficulties to obtain the complete serial section it is very difficult at present time to deduce true three dimentional patterns from the electron micrographs. The author is assuming that the three dimentional structure of this layer is the thin film of the epithelial cells which form a delicate net-like structure.

Small interruption of the epithelial cell:-It is widely recognized that except for occasional small interruptions, the endothelial layer of the endothelial cytoplasm completely lines the inner side of the basement membrane. Pease called these interruption as "pores". However, he was uncertain whether or not the pores were genuine structure or fixation artifacts, "for in life a thin film of watery cytoplasm might span these gaps." In a recent report of glomerular structure was revealed by electron microscopy, Rinehart described these interruption as small segments of cytoplasm devoid of fluid or cytoplasmic vesicles where the surface borders is not visible. This interruption in the range of $200 \mathrm{~m} \mu$ was confirmed in this study. The author assumed this interruption as a physiological interruption, and this is neither "watery cytoplasm" nor "cytoplasmic vesicle." The fact that, in an electron microscopic study on ascites hepatoma (Takamatsu, unpublished), the delicate cytoplasmic process were fairly demonstrated, indicates that when tissues were immersed in buffered osmium, little tendency was observed for their morphological characteristics to 
undergo any alteration. The fact that mentioned above is able to neglect the "artifact". Thus the interruption of the endothelial layer assumed to be a physiological interruption. The three dimentional structure of this layer is considered to be a fenestrated cytoplasmic film of the epithelial cells line completely inner side of the basement membrane.

The nomenclature of the fine structure of the glomerular tuft as follows will be afford facillities for the further studies of the glomerular structure.

\section{NOMENCLATURE OF THE FINE STRUCTURE OF THE} NORMAL GLOMERULUS

1) "Process-like structure" or "Process"

Although the process-like structures are the cytoplasmic processes of the epithelial cell, nomenclature was given to them from the shape showing in the section of the electron micrograph.

2) "Epithelial cell layer"

The layer in which innumerable process-like structures are demonstrated.

3) "Interprocess-pore"

Spaces exists between each two processes with light density.

4) "Outer translucent layer"

Lightly densitic layer which exist between the "epithelial layer" and the "basement membrane".

5) "Basement membrane"

Highly densitic middle layer of the glomerular wall.

6) "Endothelial layer"

Light densitic double-line structure which exists inner aspect of the basement membrane.

7) "Endothelial pore"

Interruptions of the endothelial layer.

8) "Inner translucent layer"

Electric translucent layer which exist between the "basement membrane" and the "endothelial layer".

9) "Mesangium" and "Mesangiumcell"

\section{SUMMARY}

Comparative study of the normal glomerulus by the specific silver impregnation method and with electron microscope were performed.

1) The basement membrane, having thickness of $100 \mathrm{~m} \mu$, is a continuous layer which is related to the overlying epithelium. No fibrillar structure were 
found in it.

2) There is no basement membrane between the "mesangium" and the capillary lumina. This finding was confirmed both by specific silver impregnation method and with electron microscope.

3) Points requiring some further study about the mesangium.

4) The epithelial layer, which is a cytoplasmic film of the epithelial cell overlying the basement membrane, is demonstrated as the "process-like structure" in section. The author also discussed about the three dimentional structure of this layer.

5) The endothelial cell and endothelial layer were observed inner side of the basement membrane. The endothelial layer might have the interruption in physiological condition. Thus, the endothelial layer is assumed to be a fenestrated thin film of the cytoplasm of the epithelial cell.

6) Nomenclature of the fine structure of the normal glomerulus was given.

The author wish to express his gratitude to Prof. Tadayoshi Kobayashi, Dr. Yonosuke Watanabe for suggesting this investigation as well as for constant guidance in the course of work. Thanks are also due to Prof. Yoshio Kusama for reading and revising the manuscript.

\section{REFERENCES}

1. Allen, R. B.: Anat. rec. 35: 30, 1927.

2. Bargmann, W.: Zeitschr. f. Zellforsch. u. mikr. Anat. 14: 73, 1931.

3. Bargmann, W.: Zeitschr, f. Zellforsch. u. mikr. Anat. 8: 765, 1929.

4. Borst, J. G.: Zeitschr. mikr.-anat. Forsch. 23: 455, 1931.

5. Gross, W.: Beitr. path. Anat. 65: 387, 1919.

6. Jones, D. B.: Am. J. of Path. 27: 6, 991, 1951.

7. Jones, D. B.: Am. J. of Path. 29: 1, 33, 1953.

8. Jones, D. B.: Am. J. of Path. 29: 4, 619, 1953.

9. Krauspe, C.: Virch, Arch. 237: 475, 1932.

10. McGregor, L.: Am. J. of Path. 5: 6, 545, 1929.

11. Möllendorff, W.: Zeitschr. f. Zellforsch. u. mikr-Anat. 6: 441, 1927.

12. Ribbert, H.: Arch. mikr. Anat. 17: 113, 1880.

13. Volterra, M.: Zeitschr. f. Zellforsch. 7: 135, 1928.

14. Zimmermann, K. W.: Zeitschr. f. mikr-anat. Forsch. 32: 176, 1933.

15. Dalton, A. J.: J. Nat. Cancer. Inst. 11: 1163, 1951.

16. Hall, B. V.: Anat. Rec. 118: 425, 1954.

17. Pease, D. C. \& Baker, R. F.: Am. J. Anat. 87: 349, 1950.

18. Rinehart, J. R., Farquhar, M. G. \& Haw Chan Jung: Am. J. of Path. 29: 21, 1953.

19. Rinehart, J. R.: Arch. of Path. 59: 439, 1955.

20. Sjöstrand, F. S. \& Rhodin, J.: Exper. cell research. 4: 2, 426, 1954. 

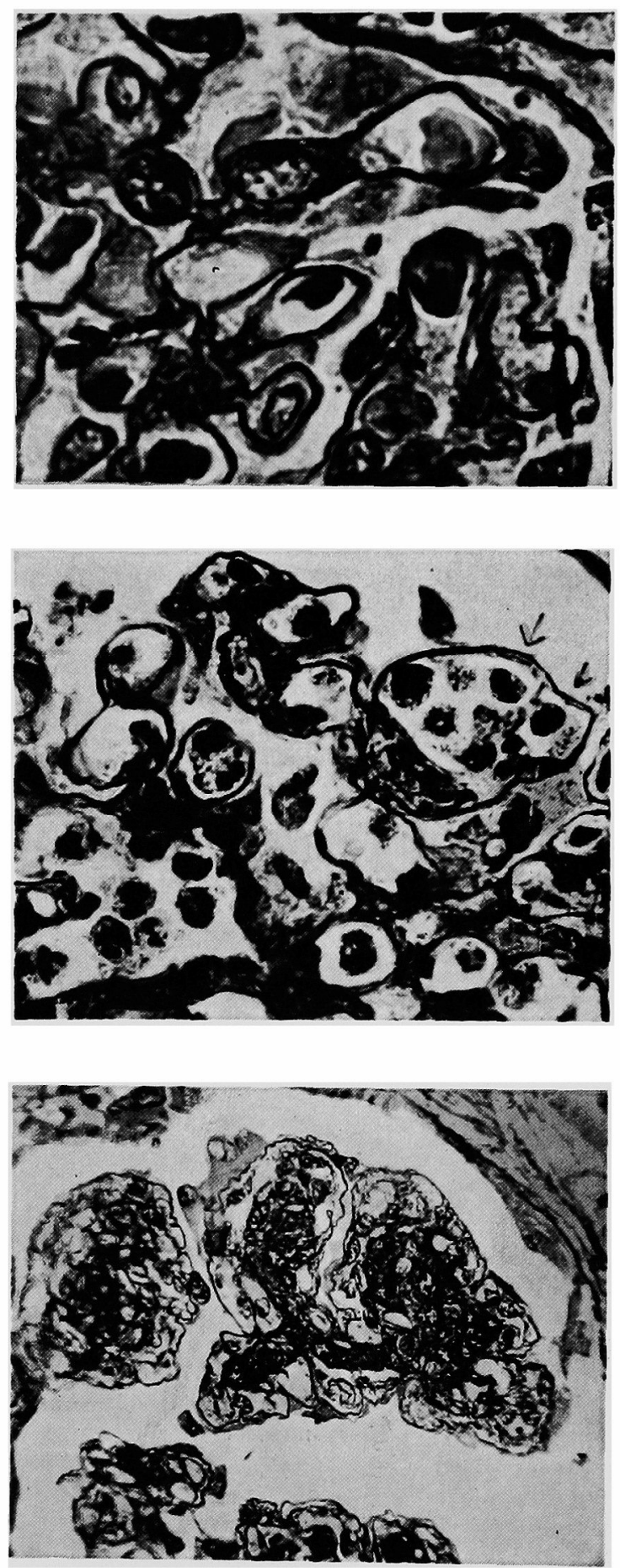

Fig. 1 New-born kidney, normal

There is no argylophil fibrils between the capillary lumina and the mesangium.

Fig. 2 Glomerulitis

Some glomrular basement membrane divided into two or three rather delicate argylophil fibrils.

Fig. 3 Subacute glomerulonephritis

Remarkable increasing of mesangium. 

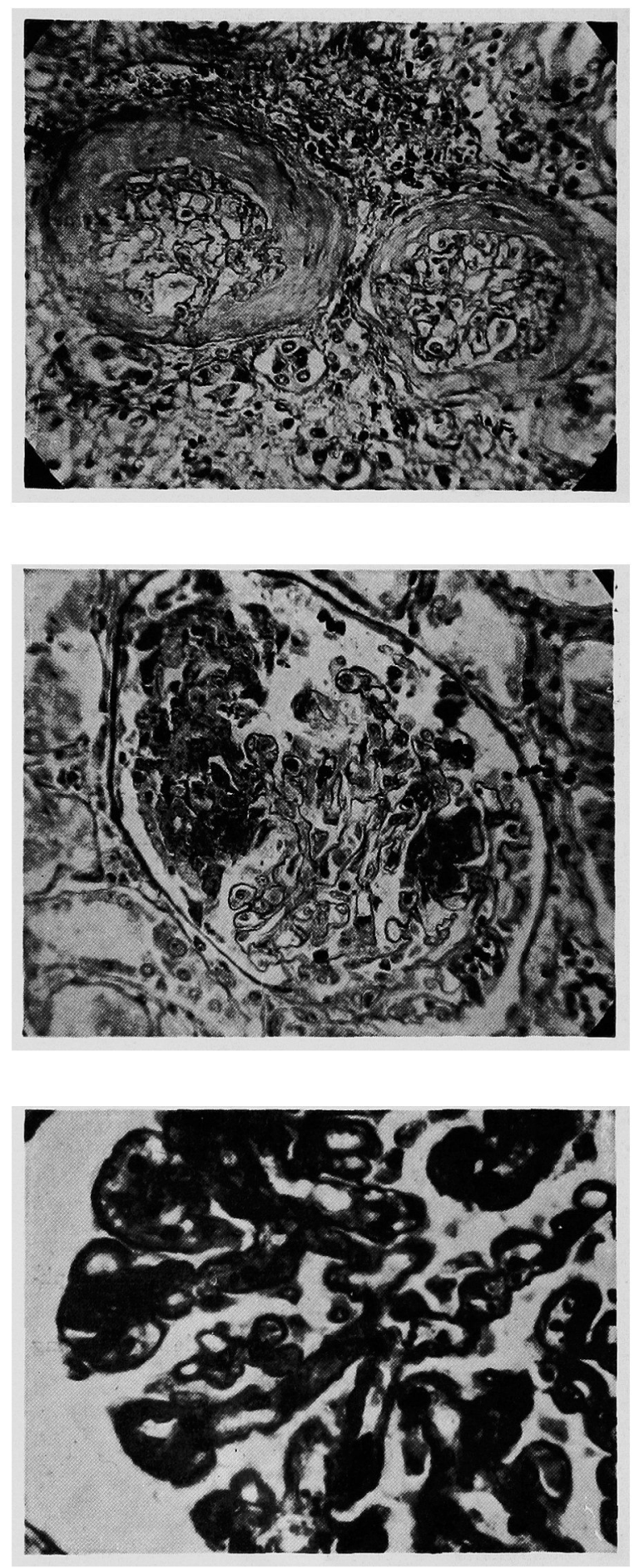

Fig. 4 Chronic glomerulonephritis Thick hyaline crescent and glomerular collaps.

Fig. 5 Embolic focal glomerulonephritis

Fragmentaion of the basement membrane.

Fig. 6 Wire-loop lesion in disseminated lupus erythema. tosus

Deposition of hyaline material in glomerular tufts. 


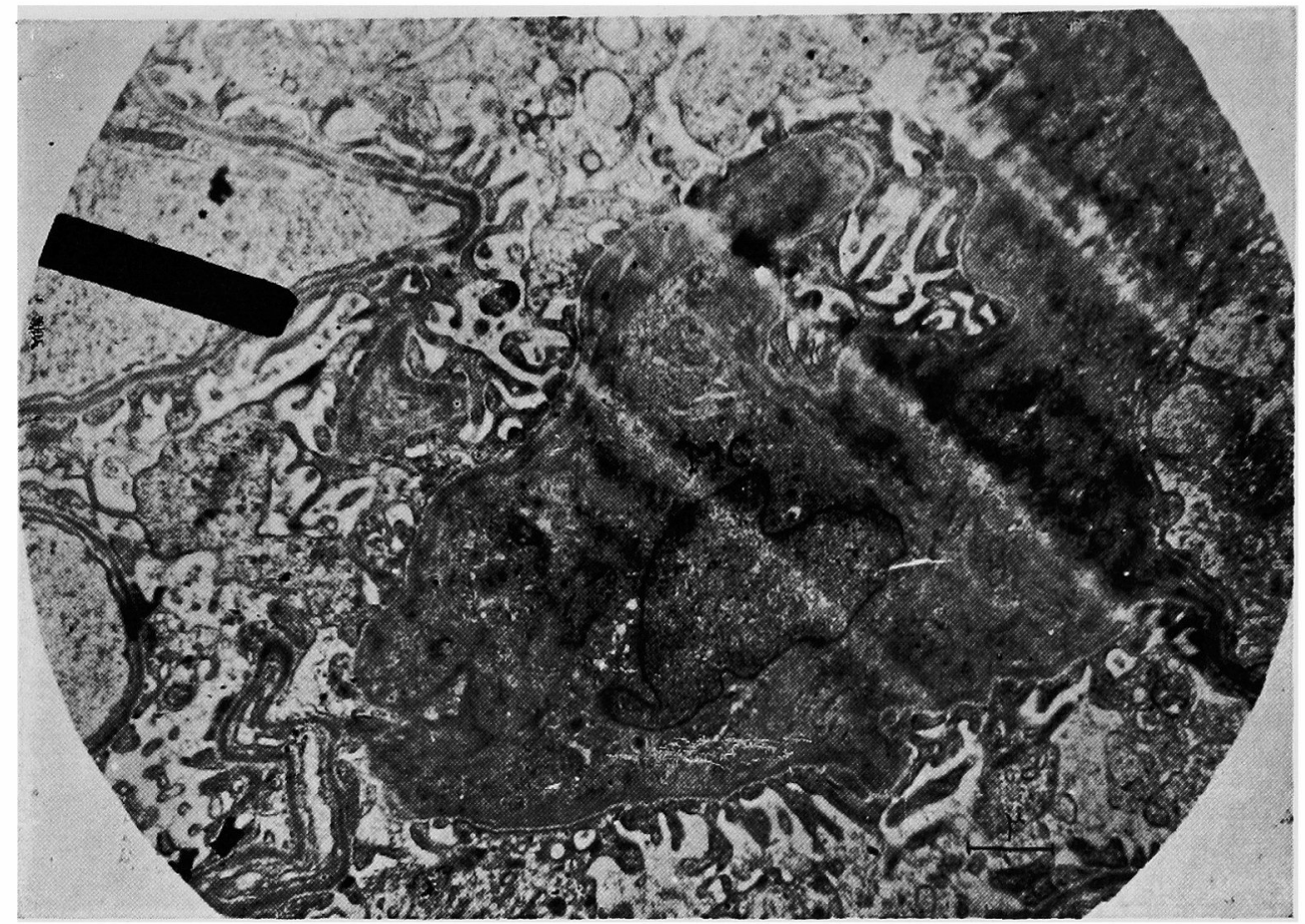

Fig. 7 MC : Mesangium cell

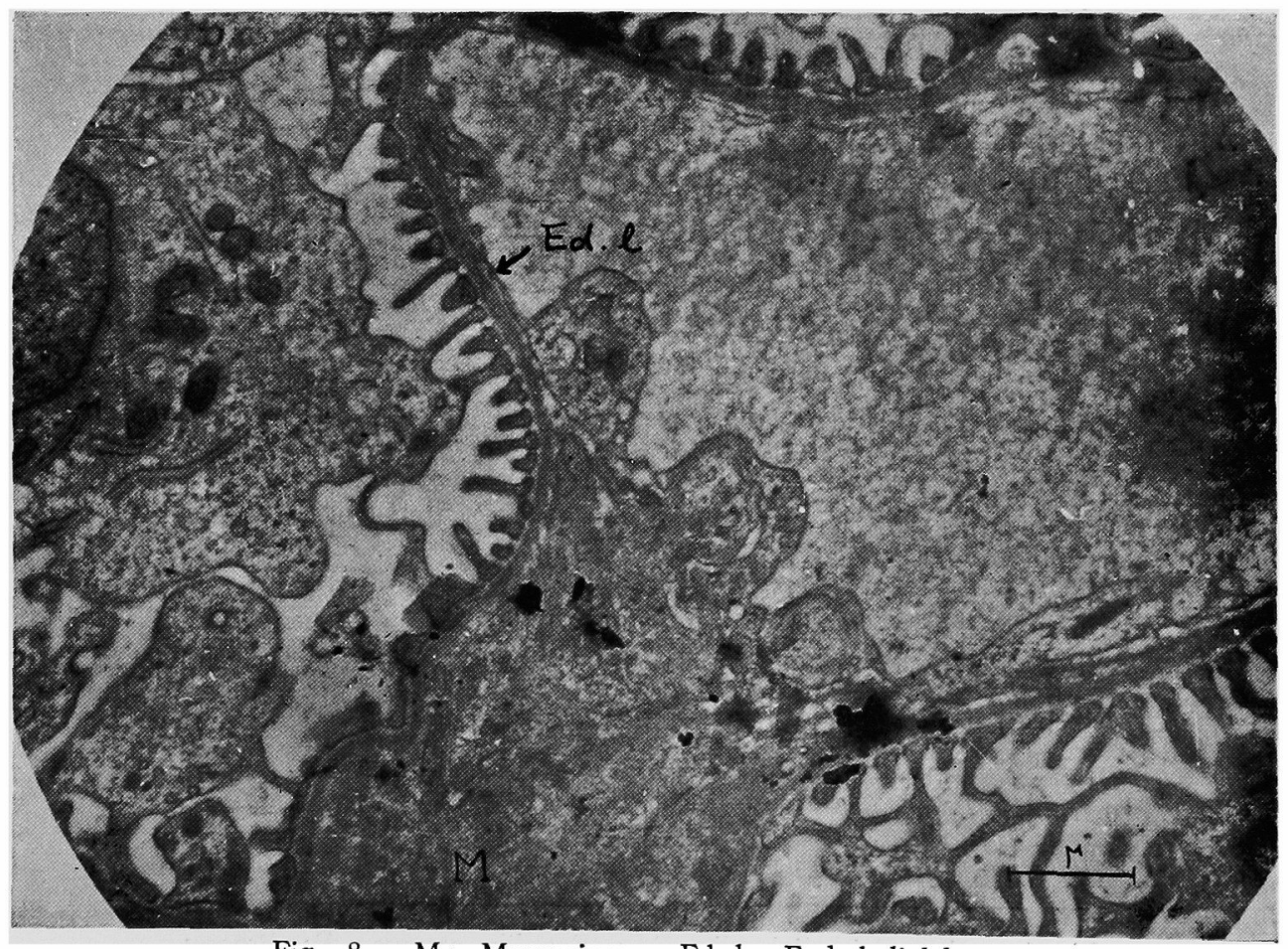

Fig. $8 \quad \mathrm{M}$ : Mesangium Ed. 1: Endothelial layer 


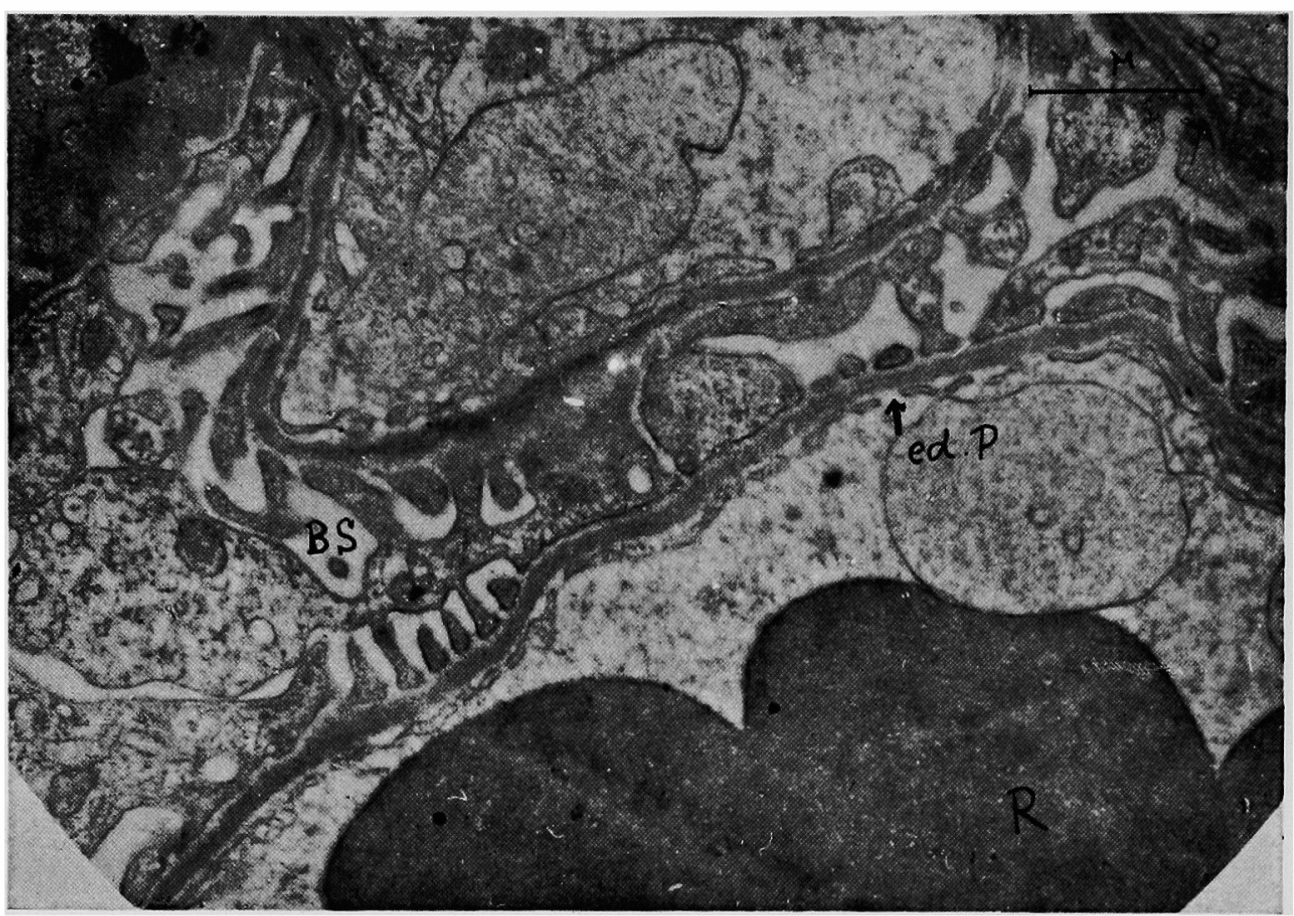

Fig. 9 ed. P.: Endothelial pore R: Red blood cell BS: Bowman's space

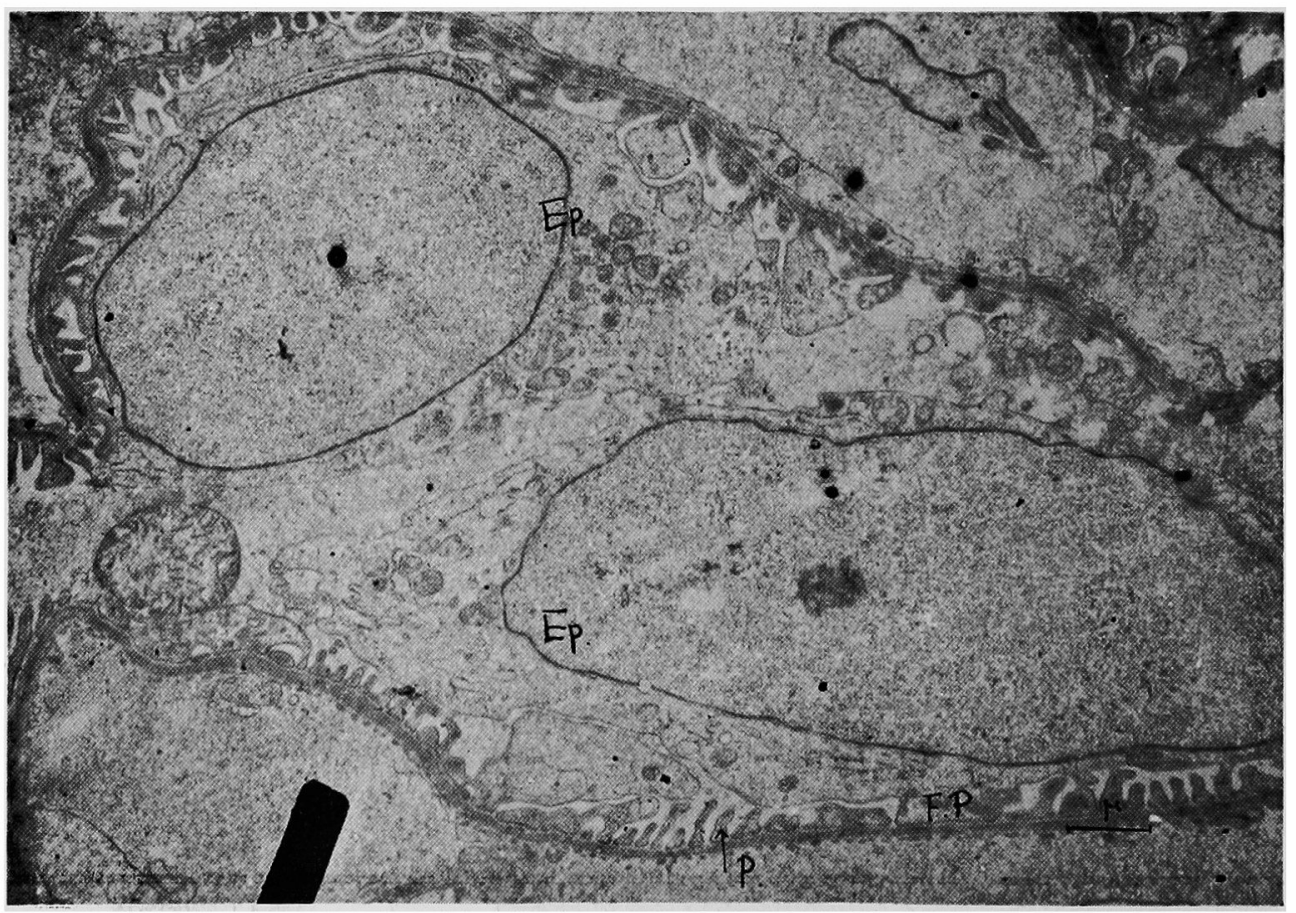

Fig. 10 Ep: Epithelial cell F.P: Foot-like process P: Process 


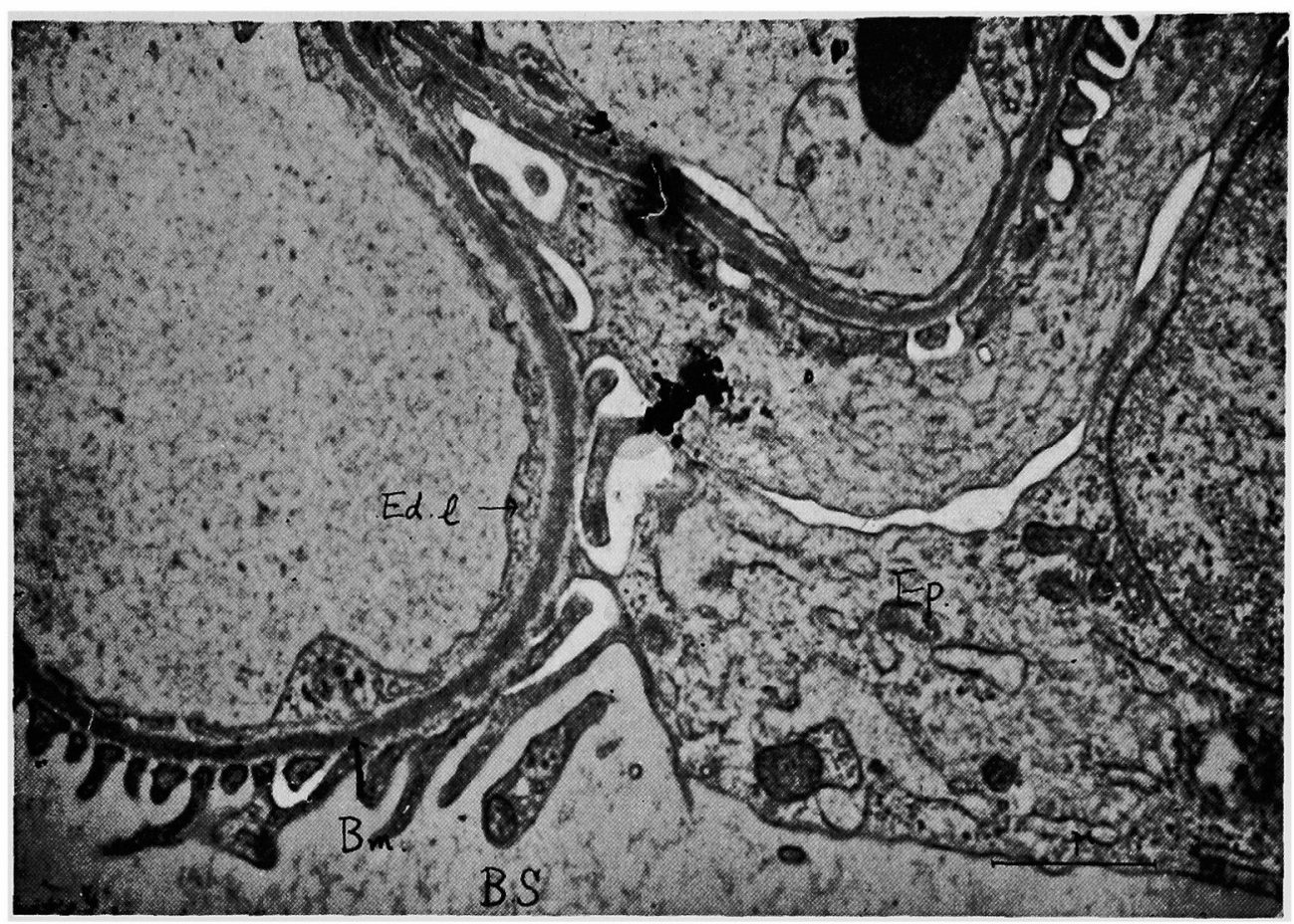

Fig. 11

Bm: Basement membrane Ep: Epithelial cell Ed.1: Endothelial layer B.S: Bowman's space

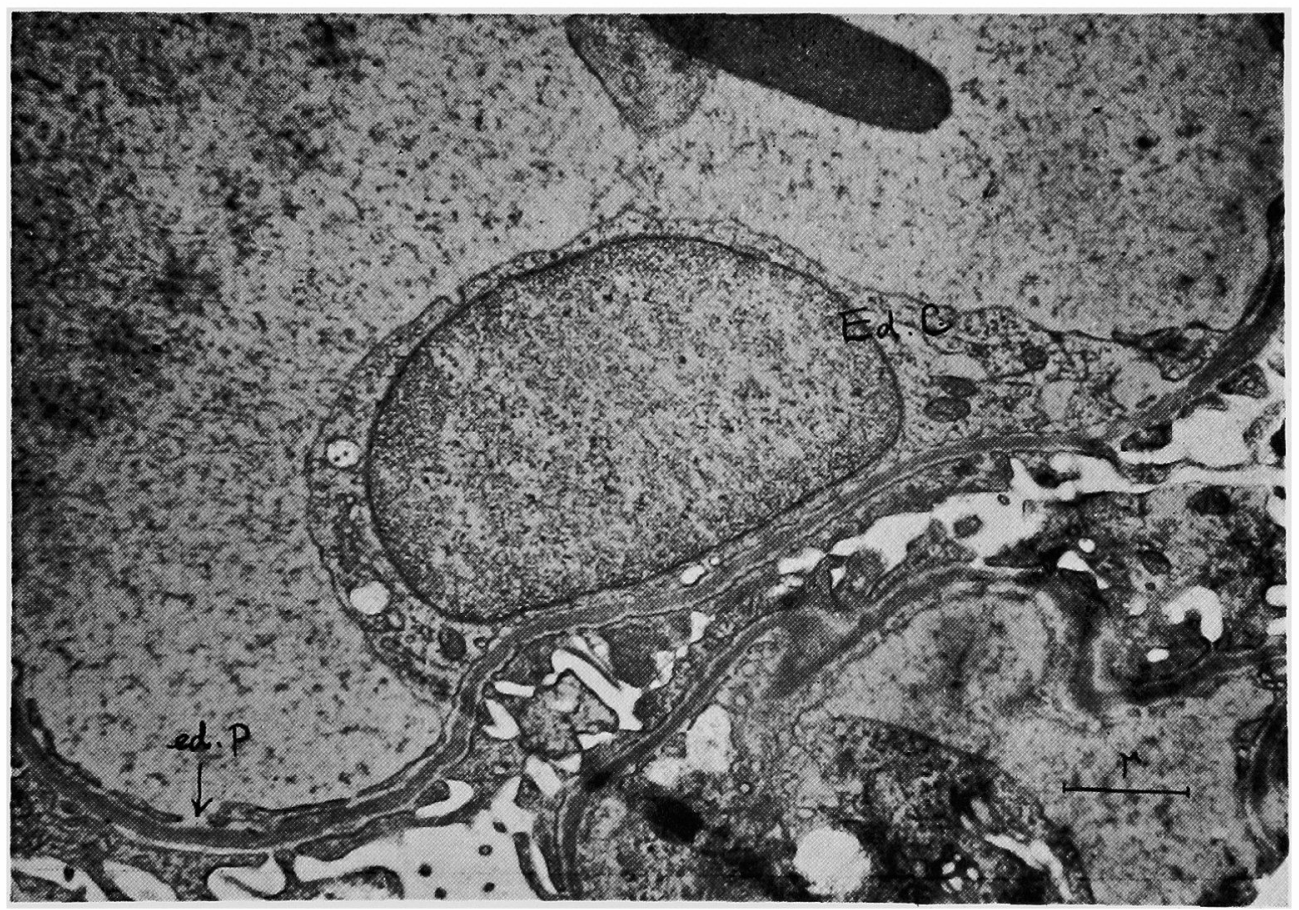

Fig. 12 Ed.C: Endothelial cell ed.P: Endothelial pore 